CERN LIBRARIES, GENEVA

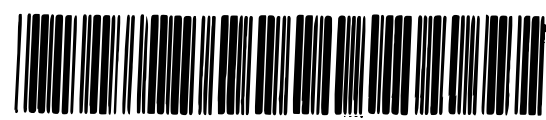

CM-P00064357

EUROPEAN ORGANIZATION FOR NUCLEAR RESEARCH

CERN ISR-BOM/79-15

A PROJECT TO OPERATE THE CERN INTERSECTING STORAGE RINGS (ISR)

WITH ANTIPROTONS

P.J. Bryant

Paper presented at the 1979 Particle Accelerator Conference, San Francisco, March 12 - 14, 1979

Geneva, Switzerland

March 1979 


\section{P.J. Bryant}

\section{Abstract}

The storage of antiprotons in the ISR for proton antiproton physics is planned for 1981, using the $3.5 \mathrm{GeV} / \mathrm{c}$, cooled, antiproton source, which is currently being built at CERN. Two schemes have been studied for achieving this aim. Firstly, the antiprotons could be injected at $3.5 \mathrm{GeV} / \mathrm{c}$ from the cooling ring and accelerated in the ISR or secondly, they could be injected into the ISR at $26 \mathrm{GeV} / \mathrm{c}$ after acceleration in the CERN Proton Synchrotron (PS). Although the latter scheme is more expensive, since it requires a new transfer line, it has been adopted as it is operationally more reliable and as it makes it possible to stack the antiprotons. With five full-intensity pulses, the circulating antiproton current would be $0.15 \mathrm{~A}$, which, with a $30 \mathrm{~A}$ proton beam, would yield a luminosity of $1.3 \times 10^{29} \mathrm{~cm}^{-2} \mathrm{~s}^{-1}$ in a standard intersection and $9.2 \times 10^{29} \mathrm{~cm}^{-2} \mathrm{~s}^{-1}$ with the planned superconducting low- $\beta$ scheme.

\section{Introduction}

The storage of antiprotons in the ISR was first discussed in 1962 by K. Johnsen ${ }^{1}$ before the ISR were constructed. The idea was to collect antiprotons generated in a target by an ejected beam from the CERN-PS. Later, it was proposed to use antiprotons created from antihyperon decay ${ }^{2} 3$. With the advent of the CERN $300 \mathrm{GeV} / \mathrm{c}$ Proton Synchrotron (SPS), a study was based on the use of a $200 \mathrm{GeV} / \mathrm{c}$ beam to produce the secondary antiprotons for the ISR either directly from a target or via antihyperon decay ${ }^{4}$. The estimated luminosity was in the range $10^{25}$ to $10^{26} \mathrm{~cm}^{-2} \mathrm{~s}^{-1}$. The cooling of the antiprotons using an electron beam in a separate device is mentioned in this reference but not considered. The problem was re-examined in $1975^{5}$, this time with a $400 \mathrm{GeV} / \mathrm{c}$ primary beam. Electron cooling was again mentioned but not considered. Soon after, the possibility of electron cooling for antiproton beams produced from PS protons was studied 6 , but this was quickly followed by schemes based on stochastic cooling either in the ISR itself or in a separate ring ${ }^{7}$. For the latter case, a luminosity of $7 \times 10^{26} \mathrm{~cm}^{-2} \mathrm{~s}^{-1}$ was estimated. Stochastic betatron cooling was first suggested by $\mathrm{S}$. Van der Meer ${ }^{8}$ and stochastic momentum cooling is believed to have been first suggested by R.B. Palmer of Brookhaven National Laboratory during the 1975 ISABELLE Summer Study ${ }^{9}$. It was quickly recognized that, in order to achieve useful luminosities for physics, special cooling schemes would be essential and considerable work was carried out by the ISR Division in this field ${ }^{10}$. By this time, the idea of a separate accumulator ring operating with stochastic cooling at 3 to $4 \mathrm{GeV} / \mathrm{c}$ and using antiprotons made by PS protons on a target was well established. Achieving luminosities in the range $10^{29}$ to $10^{30} \mathrm{~cm}^{-2} \mathrm{~s}^{-1}$ was thought to be possible ${ }^{11}$. The emphasis then changed to using antiprotons in the SPS 12 following the work of C. Rubbia13,14 and the "Initial Cooling Experiment" (ICE) was set up in 1977 as a precursor of the Antiproton Accumulator 15 which is currently under construction. Initially, it was foreseen to transfer the antiprotons from the AA ring to the SPS or the ISR at $3.5 \mathrm{GeV} / \mathrm{c}$. However, for SPS operation, this scheme was abandoned in favour of having post-acceleration in the PS to $26 \mathrm{GeV} / \mathrm{c}^{16}$. Similarly, for ISR operation there are advantages in having post-acceleration to $26 \mathrm{GeV} / \mathrm{c}$ and following a final project study17, the proposal to build a new transfer line allowing $26 \mathrm{GeV} / \mathrm{c}$ injection was approved at the end of 1978. Although the $3.5 \mathrm{GeV} / \mathrm{c}$ injection option has been replaced by that for $26 \mathrm{GeV} / \mathrm{c}$, it is briefly considered here since if it were to be revived, it would be possible to have antiproton-antiproton collisions in the ISR.

\section{2. $3.5 \mathrm{GeV} / \mathrm{c}$ Injection Option}

Figure 1 shows the CERN site with the AA ring and the new transfer tunnels which are currently under construction. Originally, it was proposed to eject the antiprotons from the AA ring along TT2 into Ring 1 of the ISR at $3.5 \mathrm{GeV} / \mathrm{c}$ (dashed line in Fig. 1). For this mode of operation, the ISR must accelerate the antiprotons to their final energy in the range 11 to $31 \mathrm{GeV} / \mathrm{c}$, which requires crossing transition. Not only would this have required a specially designed and installed transition jump, but it would have prevented the ISR from accelerating more than a single pulse of $30 \mathrm{~mA}$, since no way is known for getting a coasting stack across transition. It would also raise problems of compatibility with low- $\beta$ insertions and the question of whether it is better to ramp experimental magnets, such as the SplitField Magnet, which has a 900 ton solid core, during the acceleration or to wait until the final energy is reached. When studied in detail 17 , these problems do not turn out to be impossible to solve but their concurrence leads to a long and complicated setting up procedure with poor reliability.

\section{3. $26 \mathrm{GeV} / \mathrm{c}$ Injection Option}

For post-acceleration, the antiprotons are returned to the PS via TTL2 and TT2 (Fig. 1). After acceleration, the particles can either be ejected to the SPS via a new line TT70 or to the ISR via a new line TT6 and the existing line TTl. Thus, the ISR is relieved of the task of accelerating the beam from $3.5 \mathrm{GeV} / \mathrm{c}$ for which it is manifëstly ill-suited and instead it is possible to exploit its excellent storage capabilities and stack antiproton pulses over several days so giving far higher luminosities than were possible with the $3.5 \mathrm{GeV} / \mathrm{c}$ injection scheme. These considerations led to the adoption of the $26 \mathrm{GeV} / \mathrm{c}$ injection scheme despite $i \cdot t s$ higher cost and it is hoped, using this scheme, to have antiprotons circulating in the ISR in 1981.

\section{Antiproton Operation and Estimated Luminosities}

One important difference from normal operation will be the rarity of antiprotons. It will take $24 \mathrm{~h}$ of continuous accumulation to reach the design figure of $6 \times 10^{11}$ antiprotons in the AA ring, which gives only $30 \mathrm{~mA}$ of circulating beam in the ISR. Thus, there will be a high premium on accurate and efficient steering and diagnostics in the transfer lines in order to avoid losses. TT2 and TTL2 can be tuned with protons travelling in the opposite direction to the antiprotons. This also applies to TT70 which can be tuned with protons ejected from the SPS, but the TT6 line can only be tuned with antiprotons and specially sensitive electromagnetic pick-ups will be installed in order to do this with low-intensity pre-pulses from the AA ring.

Somewhat arbitrarily, an operation cycle of stacking five pulses of $6 \times 10^{11}$ antiprotons over four days followed by six days of stable beam conditions has been adopted (Fig. 2). The initial setting up time has been estimated at $8 \frac{1}{2} \mathrm{~h}$ after which the beams are available for physics except for brief interruptions of $1 \mathrm{~h}$ for the injection of new pulses.

Table 1 lists the peak and integrated luminosities in a standard ISR intersection and in the conventional 


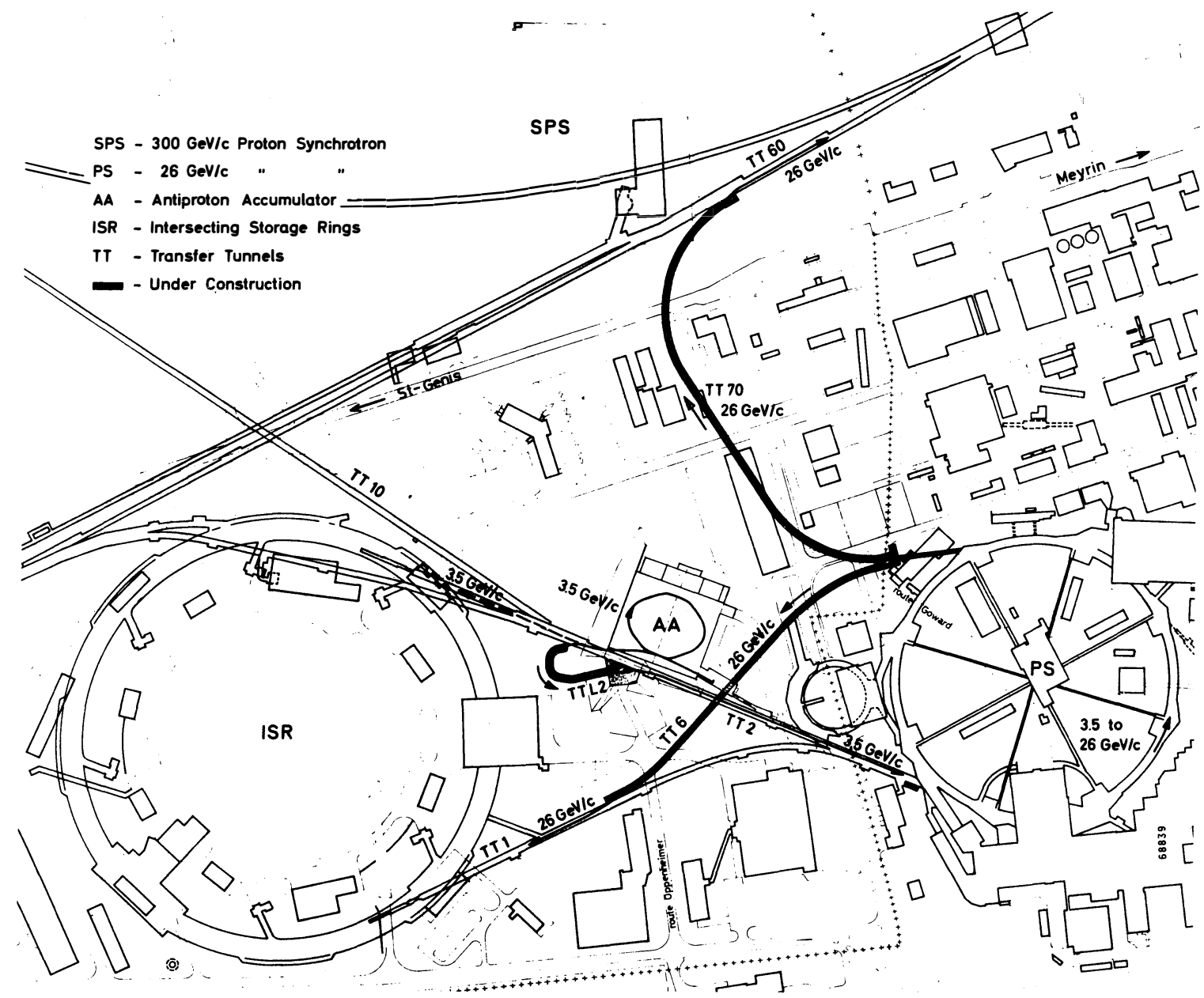

Fig. 1 The Transfer of Antiprotons Between the CERN Machines

and superconducting $10 w^{-} \beta$ schemes assuming a 30 A proton beam, $100 \%$ survival and zero transverse emittance blow-up during transfer from the AA ring to the ISR and no stochastic cooling for either beam in the ISR.

Table 1 is based on $26 \mathrm{GeV} / \mathrm{c}$ operation but operation at lower energies will be possible with the same operation cycle (Fig. 2). For the maximum ISR energy of

$31.4 \mathrm{GeV} / \mathrm{c}$, the stacking over the first four days will be at $26 \mathrm{GeV} / \mathrm{c}$ and only once the stacking has been completed will the coasting beams be accelerated by phase displacement to $31.4 \mathrm{GeV} / \mathrm{c}$.

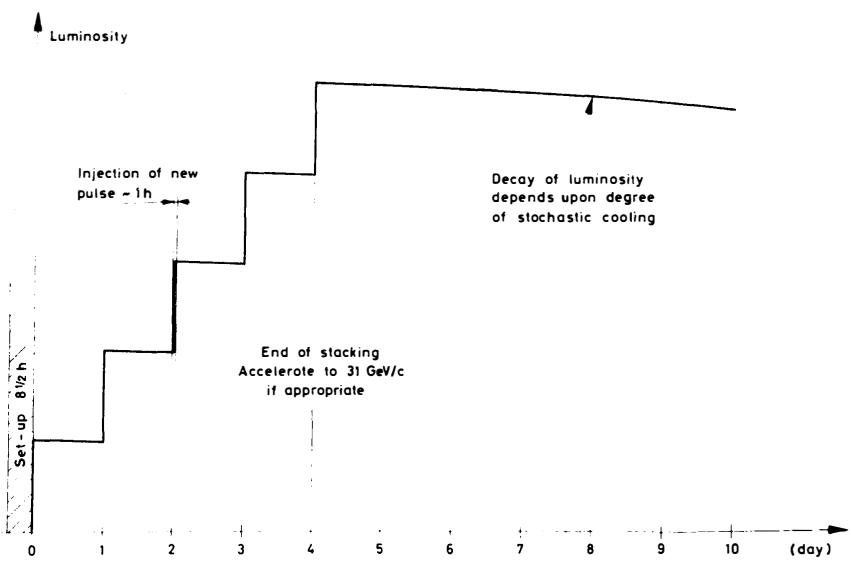

Fig. 2 Luminosity Profile During a 10-Day Physics Run With Stacking During the First 4 Days
TABLE 1

ISR Performance at $26.6 \mathrm{GeV} / \mathrm{c}$ Conditions: $\mathrm{I}_{\mathrm{p}}=30 \mathrm{~A}, \mathrm{I}_{\overline{\mathrm{p}}, \max }=0.15 \mathrm{~A}$

\begin{tabular}{|c|c|c|c|}
\hline$\overbrace{\text { Parameter }}^{\text {Inter }}$ & $\begin{array}{l}\text { Standard } \\
\beta_{\mathrm{v}}=14 \mathrm{~m}\end{array}$ & $\begin{array}{c}\text { Steel } 10 \mathrm{w}-\beta \\
\beta_{\mathrm{v}}=3 \mathrm{~m}\end{array}$ & $\begin{array}{l}\text { SC } 10 \mathrm{w}-\beta \\
\beta_{\mathrm{v}}=0.3 \mathrm{~m}\end{array}$ \\
\hline$[\mathrm{mm}]$ & 1.18 & 0.55 & 0.17 \\
\hline$[\mathrm{mm}]$ & 0.68 & 0.31 & 0.10 \\
\hline$h_{\text {eff }}$ & 3.4 & 1.6 & 0.5 \\
\hline$\left[\mathrm{cm}^{-2} \mathrm{~s}^{-1}\right]$ & $1.3 \times 10^{29}$ & $2.8 \times 10^{29}$ & $9.2 \times 10^{29}$ \\
\hline$\frac{1}{\mathrm{~L}} \frac{\mathrm{dL}}{\mathrm{dt}} \quad\left[\mathrm{s}^{-1}\right]$ & $1.4 \times 10^{-6}$ & $1.4 \times 10^{-6}$ & $1.4 \times 10^{-6}$ \\
\hline $\int_{(10 \text { days })} \mathrm{Ldt}\left[\mathrm{cm}^{-2}\right]$ & $5.7 \times 10^{34}$ & $1.2 \times 10^{35}$ & $4.0 \times 10^{35}$ \\
\hline
\end{tabular}

\section{Use of Stochastic Cooling in the ISR}

It has been tacitly assumed above that it is best to collide the antiprotons with the highest possible intensity proton beam. However, for many experiments, a small loss in luminosity would be readily accepted if the current loss rates and hence the background could be reduced to zero. Stochastic cooling offers this possibility and furthermore the integrated luminosity may not in fact be lower than for the high-intensity, non- 
cooled case. In order to evaluate the potential gain, some conservative assumptions were made and the highest proton beam current was computed ${ }^{18}$ at which a somewhat improved version of the existing experimental equipment for cooling in the ISR would balance the beam blow-up due to intra-beam scattering, gas scattering and coupling for a vertical emittance equal to that of the incoming antiproton beam. The antiproton beam is also assumed to have its emittance maintained constant by cooling. The results of these calculations are given in Table 2 .

\section{TABLE 2}

Estimated $26 \mathrm{GeV} / \mathrm{c}$ Performance with Cooled Beams Conditions: $\mathrm{I}_{\mathrm{p}}=7.25 \mathrm{~A}, \mathrm{I}_{\overline{\mathrm{p}}, \max }=0.15 \mathrm{~A}$ with cooling

\begin{tabular}{|c|c|c|c|}
\hline$\underbrace{\text { Int }}_{\text {Parameter }}$ & $\begin{array}{l}\text { St and ard } \\
\beta_{\mathrm{v}}=14 \mathrm{~m}\end{array}$ & $\begin{array}{l}\text { Steel low }-\beta \\
\beta_{\mathrm{v}}=3 \mathrm{~m}\end{array}$ & $\begin{array}{l}\text { SC } 10 \mathrm{w}-\beta \\
\beta_{\mathrm{v}}=0.3 \mathrm{~m}\end{array}$ \\
\hline$[\mathrm{mm}]$ & 0.68 & 0.31 & 0.10 \\
\hline$[\mathrm{mm}]$ & 0.68 & 0.31 & 0.10 \\
\hline$h_{\text {eff }}$ & 2.4 & 1.1 & 0.35 \\
\hline$\left[\mathrm{cm}^{-2} \mathrm{~s}^{-1}\right]$ & $4.5 \times 10^{28}$ & $9.9 \times 10^{28}$ & $3.1 \times 10^{29}$ \\
\hline$\frac{1}{\mathrm{~L}} \frac{\mathrm{dL}}{\mathrm{dt}} \quad\left[\mathrm{s}^{-1}\right]$ & 0 & 0 & 0 \\
\hline $\int_{(10 \text { days })} \mathrm{L} \mathrm{dt}\left[\mathrm{cm}^{-2}\right]$ & $3.1 \times 10^{34}$ & $6.8 \times 10^{34}$ & $2.1 \times 10^{35}$ \\
\hline
\end{tabular}

Symbols Used in Tables.

$\sigma \quad:=$ standard deviation of the vertical particle distribution,

$$
\begin{aligned}
& h_{\text {eff }}=\sqrt{2 \pi} \sqrt{\sigma_{\mathrm{p}}^{2}+\sigma_{\overline{\mathrm{p}}}^{2}}, \\
& \mathrm{~L} \quad=\text { luminosity, } \\
& \mathrm{I}=\text { current, } \\
& \mathrm{p} \overline{\mathrm{p}}=\text { proton or antiproton parameters, } \\
& \beta_{\mathrm{v}} \quad=\text { vertical betatron amplitude function, } \\
& \mathrm{t} \quad=\text { time. }
\end{aligned}
$$

\section{Experimental Magnets}

The Split-Field Magnet spectrometer, the Superconducting Solenoid and the Open Axial Field Magnet substantially affect the circulating beams and since they are common to both beams, their fields cannot be simply reversed for antiproton operation. In the case of the Split-Field Magnet, this has made it necessary to accept a reduced horizontal aperture for the antiproton beam. The effect of the Superconducting Solenoid will be to separate the two beams vertically by $\sim 17 \mathrm{~mm}$. This will be corrected by adding vertical orbit bumps of $\pm 8.5 \mathrm{~mm}$ to each beam, for which new dipoles will be needed. Fortunately, the Open Axial Field Magnet has far less influence and the existing vertical orbit correctors are sufficient to bring the beams into head-on collision. All other experimental magnets can be used without any special considerations.

\section{Acknowledgements}

The author would firstly like to thank the ISR Division as a whole for having entrusted him with the task of reporting their work and then to thank, on behalf of the ISR Division, the CERN Directorate and the SPS and PS Divisions for all of their help and encouragement.

\section{References}

1. K. Johnsen, The possibility of $p+\bar{p}$ colliding beam experiments in a set of storage rings. Int. Report CERN AR/Int. SG/62-11 (1962).

2. G.K. O'Neill, Colliding beam techniques, PrincetonPennsylvania Accelerator Report PPAD 415D (1961).

3. S. Van der Meer, Filling the ISR with antiprotons from antihyperon decay, Div. Report CERN ISR-PO/70-5 (1970).

4. K. Hübner, The storage of antiprotons in the ISR, Div. Report CERN ISR-TH/73-19 (1973).

5. K. Hübner, K. Johnsen, G. Kantardjian, The feasibility of antiprotons in the ISR, Div. Report CERN ISR-LTD/75-45 (1975).

6. G.I. Budker, N. Dikansky, D. Möh1, A.N. Skrinsky, P. Strolin, L. Thorndahl, Possibilities for antiproton beams at CERN using cooling by electrons, Int. Report CERN EP/76-03 (1976).

7. D. Möhl, P. Strolin, L. Thorndahl, Stochastic cooling of antiprotons for ISR physics, Int. Report CERN EP/76-05 (1976).

8. S. Van der Meer, Stochastic damping of betatron oscillations in the ISR, Div. Report CERN ISR-P0/72-31 (1972).

9. H.G. Hereward and W. Schnel1, Private communication.

10. G. Carron, L. Faltin, W. Schnel1, L. Thorndahl, Stochastic cooling of betatron oscillations and momentum spread, Proc. Vth All-Union Conf. on Particle Accelerators, Dubna, USSR (October 1976).

11. K. Hübner, D. Möh1, P. Strolin, L. Thorndahl, Estimates of ISR luminosities with cooled beams, ISR Workshop/F-4, PS/DL/Note 76-27 (1976).

12. F. Bonaudi, S. Van der Meer, B. Pope, Antiprotons in the SPS, Parts I and II, Div. Report CERN/DG-2 (1977).

13. C. Rubbia, Unconventional use of the SPS and FNAL rings .... Seminar given at CERN (February 1976).

14. C. Rubbia, On the feasibility of $\bar{p}-p$ collisions in the SPS, Div. Report CERN EP/77-1 (1977).

15. Design study of a proton-antiproton colliding beam facility, Div. Report CERN PS/AA 78-3 (1978).

16. SPS and AC Groups, Beam transfers and modifications to the SPS accelerator for the proton-antiproton colliding beam project, Div. Report CERN SPS/AC 78-10 (1978).

17. Compiled by P.J. Bryant, ISR operation with antiprotons, Div. Report CERN ISR-BOM/78-18 (1978).

18. G. Guignard, Private communication. 